\title{
A Model for the Optical Absorption in Porous Silicon
}

\author{
Shouvik.Datta * and K.L.Narasimhan \\ Solid State Electronics Group . \\ Tata Institute of Fundamental Research, Homi Bhabha Road, Colaba, Mumbai 400 005. INDIA.
}

\begin{abstract}
In this paper, we report on the optical absorption in porous silicon. We model the absorption process assuming that porous silicon is a pseudo 1D material system having a distribution of band gaps. We show that in order to explain the absorption we specifically need to invoke - (a) $\mathrm{k}$ is not conserved in optical transitions, (b) the oscillator strength of these transitions depend on the size of the nanostructure in which absorption takes place and (c) the distribution of band gaps significantly influences the optical absorption. We also show that it is not possible to extract the band gap of porous silicon from a plot of $\sqrt{\alpha \hbar \omega}$ vs $\hbar \omega$.
\end{abstract}

Keywords : absorption coefficient, porous silicon .

PACS Numbers : 78.20.c, 78.66.j, 78.40.f

*e.mail : shouvik@tifr.res.in

Typeset using REVTEX 


\section{INTRODUCTION}

The discovery of efficient photoluminescence ${ }^{(1,2)}$ from porous silicon (PS) has attracted the attention of many researchers. The photoluminesecence peak occurs at an energy greater than the band gap of c-silicon and can be tuned through the visible spectrum by changing the preparation conditions. Canham $^{(1)}$ proposed that PS is a nanostructured material and the band gap of these nanostructures is enhanced due to quantum size effects. He argued that this would account for the fact that the luminescence energy is greater than the band gap of c-silicon. Evidence for the increase in band gap was first reported by Lehmann - from optical absorption measurements ${ }^{(3)}$. Transmission electron microscopy measurements reveal that the diameter of the columnar nanostructures that make up PS depends on preparation conditions and can be as small as $2-4 \mathrm{~nm}^{(2,3)}$. PS can hence be thought of as an assembly of pseudo 1D quantum wires. The progress in this area has been the subject of many recent reviews ${ }^{(4-8)}$. In this paper we confine ourselves to the understanding of the optical absorption process in PS.

There have been many attempts to obtain the band gap of PS from optical absorption measurements ${ }^{(9-12)}$. In these measurements, the authors assume that the absorption coefficient $(\alpha)$ of PS satisfies the same relation as in 3D bulk c-silicon viz.

$$
\sqrt{\alpha \hbar \omega}=A\left(\hbar \omega-E_{g}\right)
$$

A plot of $\sqrt{\alpha \hbar \omega}$ vs $\hbar \omega$ is a straight line and the intercept on the energy axis gives the band gap $E_{g}$. The use of this equation for PS suffers from the following criticisms

1) In general ,the absorption co-efficient depends on the joint density of states (JDOS) of the material. Eq.1 is valid only when the density of states $\mathrm{g}(\mathrm{E})$ varies ${ }^{(13)}$ with energy near the band edges as $\mathrm{g}(\mathrm{E})=\mathrm{B} \sqrt{\mathrm{E}}$. If we treat PS as a $1 \mathrm{D}$ material, it would be more appropriate to write $\mathrm{g}(\mathrm{E})=\frac{B}{\sqrt{E}}$, where $\mathrm{E}$ is measured from the band edge. Clearly the JDOS in PS is expected to be different from that of bulk c-silicon. For a $1 \mathrm{D}$ system 
having a single direct band gap $E_{g}$, it is easy to show that the JDOS is proportional to $\frac{B}{\sqrt{\left(E-E_{g}\right)}}$. In such a case, a plot of $\alpha(E)$ vs E should peak at $E_{g}$. On the otherhand, for an indirect band gap material ( assuming that momentum $\mathrm{k}$ is not conserved in this pseudo 1D system during optical transition ), it is easy to show that the JDOS is a constant and independent of incident energy ( following reference (13) ). Therefore, in either case , $\sqrt{\alpha \hbar \omega}$ is not linearly related with $\hbar \omega$ at all. Clearly the use of eq. 1 to extract the band gap is not justified for PS .

2) The nano structures that make up PS have a distribution ${ }^{(2,3,8)}$ of diameters (d). If the band gap is related to quantum size effects, then it is clear that the energy upshift $\Delta E$ ( which can be written as $\Delta \mathrm{E}=\mathrm{C} / \mathrm{d}^{X}$ ) is different for each of the nanostructures that make up PS.( The value of $\mathrm{X}$ in the simplest approximation is 2 , further details of this are discussed in section III ). In PS, we are actually dealing with a heterogeneous system with a distribution of band gaps. Hence it may not be meaningful to visualize PS as having a single band gap and that this can be extracted from equation 1.

In an attempt to address these questions we have investigated the optical absorption in PS .In this paper we clarify the absorption process in PS assuming that PS has a distribution of band gaps. Section II contains the experimental results. Here we report a non - destructive way of measuring the porosity of PS thin films using transmission measurements. Section III outlines the model used for calculating optical absorption and section IV the results of our simulations. Finally we summarise our conclusions in section $\mathrm{V}$.

\section{EXPERIMENTAL RESULTS}

PS is made by electrochemical etching of $\mathrm{P}\left(\mathrm{P}^{+}\right)$c-si in a 1:1 HF : Ethanol solution at a current density between $10-50 \mathrm{~mA} / \mathrm{cm}^{2}$. Free standing samples are lifted off the substrate by increasing the current density to $>200 \mathrm{~mA} / \mathrm{cm}^{2}$. The samples are then washed in water to get rid of $\mathrm{HF}$, rinsed in n-pentane and finally dried in air on a glass 
substrate for transmission measurements .

Transmission measurements are carried out using a CVI 240 Digikrom monochromator with a tungsten lamp as a source, silicon detector and SR 530 lock -in amplifier .For some samples we also use a Cary 1756 spectrometer. We have checked that internal multiple scattering does not dominate the absorption in our samples. This is done by making two PS samples of two different thickness ( $5 \mu$ and $10 \mu$ respectively) and ensuring that the transmission curves are the same for both samples at low absorption ${ }^{(11)}$.

The absorption coefficient $\alpha(\lambda)$ as a function of wavelength $\lambda$ is obtained from the normalised transmittance $\mathrm{T}(\lambda)$ using

$$
T(\lambda)=\frac{(1-R)^{2} \exp (-\alpha(\lambda)(1-P) t)}{1-R^{2} \exp (-2 \alpha(\lambda)(1-P) t)}
$$

The reflectivity $(\mathrm{R})$ is obtained in the low $\alpha$ region using the relation $\mathrm{R}=$ $(1-\mathrm{T}) /(1+\mathrm{T}) . \mathrm{P}$ is the porosity of the layer and $\mathrm{t}$ is its thickness.

The porosity of PS is usually determined by gravimetric measurements which also destroy the sample. We now show that optical transmission experiment allows us to measure porosity in a nondestructive fashion .

The normalized transmittance ( in eq.2) for $\alpha=0$ reduces to

$$
T_{L}=\frac{(1-R)}{(1+R)} \text { or } R=\frac{1-T_{L}}{1+T_{L}}
$$

If $\mathrm{n}$ is the real part of the refractive index, then we can also write for the reflectivity ( for $\alpha=0$ ) as

$$
R=\frac{(n-1)^{2}}{(n+1)^{2}}
$$

It follows from eq.4 that the refractive index $\left(n_{P S}\right)$ of PS is

$$
n_{P S}=\frac{1+\sqrt{R}}{1-\sqrt{R}}
$$


We model PS as consisting of two media - air ( having a refractive index ( $n_{\text {air }}$ $=1))$ and crystalline silicon $\left(\mathrm{n}_{S i}=3.44\right)$. If $\mathrm{P}$ is the porosity of a sample then we can write

$$
\begin{gathered}
n_{\text {air }} P+n_{S I}(1-P)=n_{P S} \\
P=\frac{\left(n_{S I}-n_{P S}\right)}{\left(n_{S I}-1\right)}
\end{gathered}
$$

The porosity can now be obtained using equations 4 to 7 .

To check the validity of eq.7, we calculate the porosity ( using eq.7) and compare it with the corresponding values of $\mathrm{P}$ obtained gravimetrically. Figure 1 is a plot of the porosity determined from the transmission curves and the corresponding experimentally determined porosity by gravimetric measurements as reported in the literature $^{(9,14)}$. We see from figure 1 that the points nearly fit a straight line with slope one. We hence conclude that optical transmission measurements can reliably be used to obtain the porosity of porous silicon in a non-destructive fashion .

Figure 2 shows the normalized transmission data on free standing PS films made from p type ( resistivity $4 \mathrm{ohm}-\mathrm{cm}$ ) si substrate and also from $\mathrm{p}^{+} \mathrm{si}(0.01 \mathrm{ohm}-\mathrm{cm}$ ) substrate. We see from the figure that for the PS grown on $\mathrm{p}$ type si, the onset of low absorption occurs at a smaller wavelength than that for PS grown on $\mathrm{p}^{+}$si. This signifies that the p PS has a higher band gap than $\mathrm{p}^{+}$PS and is in agreement with the reported results ${ }^{(10)}$.

Figure 3 is a plot of $\log (\alpha)$ vs $\mathrm{E}$ at $300 \mathrm{~K}$ and at $100 \mathrm{~K}$. The absorption at low temperature is reduced with respect to that at room temperature. We see that the two curves exhibit a rigid shift in agreement with other results ${ }^{(10)}$. An explanation of this phenomena on the basis of our simulated results is given in section IV .

We now attempt to understand the optical absorption process in PS with the help of some model calculations and simulations, assuming that PS has a distribution of 
band gaps .

\section{A MODEL FOR ABSORPTION IN PS HAVING A DISTRIBUTION OF BAND GAPS}

We model the nanostructures that make up PS to be 1D parallelopipeds of square cross sections of side d and constant length L. Since d is typically $20-40 \AA$, the band gap of the material is enhanced due to quantum size effects . We assume that the effective band gap ( lowest energy gap ) for a particular parallelopiped is the minimum separation between the conduction band and the valence band states for quantum numbers $\mathrm{n}_{x}=1$ and $\mathrm{n}_{y}=1$. We also assume that the absorption takes place in the bulk.

The absorption coefficient $\alpha(\mathrm{E})$ for a particular value of incident energy E can be written as

$$
\alpha(E)=\int_{E_{G}<E} \rho^{J}\left(E-E_{G}\right) F\left(E_{G}\right) P\left(E_{G}\right) d E_{G}
$$

where the integration is done over all the band gaps $\left(\mathrm{E}_{G}\right)$ of the system below the incident energy E. In equation $8, \rho_{J}$ is the joint density of states, $\mathrm{F}\left(\mathrm{E}_{G}\right)$ the oscillator strength for the optical transition and $\mathrm{P}\left(\mathrm{E}_{G}\right)$ the distribution of band gaps $\mathrm{E}_{G}$ in the material. We now discuss eq.8 in some detail.

We have already pointed out in section $\mathrm{I}$, if $\mathrm{g}(\mathrm{E})=\mathrm{B} / \sqrt{E}$ then the JDOS is $\rho^{J}=A\left(E-E_{G}\right)^{-W}$ where $\mathrm{A}$ is a constant. The value of $\mathrm{W}$ depends on whether PS is a direct or an indirect band gap material. For direct band gap material $\mathrm{W}=0.5$ and for indirect band gap material $\mathrm{W}=0$.

$\mathrm{F}\left(\mathrm{E}_{G}\right)$ is the oscillator strength governing the absorption. For indirect band gap material, the oscillator strength can be enhanced for small $\mathrm{d}^{(15)}$. This happens because the overlap between the electron and hole wavefunctions in $\mathrm{k}$ space increases as a result of quantum confinement and contributes to an increase in the oscillator strength at small d $\left(\text { large } E_{G}\right)^{(16,17)}$ and can be written as $\mathrm{F}=\mathrm{f} / \mathrm{d}^{\gamma}$ where $\gamma=5$ to 6 . This manifests itself as 
the no phonon line which has been seen in the luminescence spectra of PS ${ }^{(18)}$.

The third term in eq.8 viz. $P\left(E_{G}\right)$, is the distribution of band gaps in PS. The band gap distribution is obtained by assuming a distribution of sizes for $\mathrm{d}$ and a relation governing the upshift in energy $\Delta \mathrm{E}$ with the size $\mathrm{d}$ ( due to quantum confinement ).

In our model we have considered two possible distribution of sizes. One is Gaussian and the other is Lognormal ${ }^{(19)}$ distribution given by $P^{G}(\mathrm{~d})$ and $P^{L}(\mathrm{~d})$ respectively.

$$
P^{G}(d)=\frac{1}{(\sqrt{2 \Pi \sigma})} \exp \left(-\frac{\left(d-d_{0}\right)^{2}}{2 \sigma^{2}}\right)
$$

where $d_{0}$ is the mean size and the $\sigma$ the standard deviation.

$$
P^{L}(d)=\frac{1}{\sigma_{L} d \sqrt{2 \Pi}} \exp \left[-\frac{\left(\ln (d)-m_{0}\right)^{2}}{2 \sigma_{L}{ }^{2}}\right]
$$

where $\mathrm{m}_{0}=\ln \left(\mathrm{d}_{0}\right)$, and $\sigma_{L}=\ln (\sigma), d_{0}$ being the mean size and $\sigma$ the standard deviation.

Electron microscopy measurements ${ }^{(2,3,20)}$ suggest that for $\mathrm{p}$ PS, $\mathrm{d}_{0}$ is around 30 $\stackrel{\circ}{A}$ and $\sigma \approx 4 \stackrel{\circ}{A}$. Figure 4 shows the distribution of sizes for Lognormal and Gaussian distributions for $\mathrm{d}_{0}=30 \AA$ and $\sigma=4 \AA$.

The energy up shift for the confinement $\Delta \mathrm{E}$ can be written as

$$
\Delta E=E_{G}-E_{g}=C / d^{X}
$$

where $\mathrm{E}_{g}$ is the crystalline $\mathrm{Si}$ fundamental indirect band gap $\approx 1.17 \mathrm{eV}$ and $\mathrm{E}_{G}$ is the increased band gap due to quantum confinement.

The value of $\mathrm{X}$ is 2 in the usual effective mass approximation. However, for nanocrystals, the effective mass itself becomes size dependent and the exponent $\mathrm{X}$ has been reported to vary from $1.2-1.8$ and becomes 2 at large size ${ }^{(15,21)}$. For the purpose of our simulation we consider two cases

a) $\mathrm{X}=2, \mathrm{C}=486 \mathrm{eV} / \AA^{2}\left(\mathrm{E}_{G O}=1.71 \mathrm{eV} \text { for } \mathrm{d}=30 \AA\right)^{(20,22)}$.

and 


$$
\text { b) } \mathrm{X}=1.4, \mathrm{C}=126 \mathrm{eV} / \AA^{1.4}\left(\mathrm{E}_{G O}=2.25 \mathrm{eV} \text { for } \mathrm{d}=30 \AA\right)^{(21)} \text {. }
$$

The interaction volume of the column structures with incident light is $\mathrm{V}(\mathrm{d})=\mathrm{L}$ $\mathrm{d}^{2}$. The resulting distribution $\mathrm{R}(\mathrm{d})=\mathrm{P}(\mathrm{d}) \times \mathrm{V}(\mathrm{d})$ is properly normalized to have equal number of dipole oscillators in each case. We now make a change of variable from d to $\mathrm{E}_{G}$ $\left(\mathrm{E}_{G}=\mathrm{E}_{g}+\mathrm{C} / \mathrm{d}^{X}\right)$ to get the corresponding distribution $\mathrm{P}\left(\mathrm{E}_{G}\right)$ of the band gaps.

For a Gaussian distribution of sizes (eq.9) - the distribution of band gaps is

$$
P^{G}\left(E_{G}\right)=P_{0} \int_{0}^{E_{b}}\left(E_{G}-E_{g}\right)^{(-X-3) / X} \exp \left[-A\left(\left(\frac{E_{G 0}-E_{g}}{E_{G}-E g}\right)^{1 / X}-1\right)^{2}\right] d E_{G}
$$

where $\mathrm{P}_{0}$ is a normalization constant, $\mathrm{A}=1 / 2\left(d_{0} / \sigma\right)^{2}$ and $\mathrm{E}_{b}$ is the maximum value of $\mathrm{E}_{G}$.

Following a similar procedure for the Lognormal distribution (eq.10) we have

$$
P^{L}\left(E_{G}\right)=R_{0} \int_{0}^{E_{b}}\left(E_{G}-E_{g}\right)^{(-X-2) / X)} \sigma_{L}^{-1} \exp \left[-B\left(\frac{\ln \left(C / E_{G}-E_{g}\right)^{1 / X}}{\ln \left(C / E_{G 0}-E_{g}\right)^{1 / X}}-1\right)^{2}\right] d E_{G}
$$

where $\mathrm{R}_{0}$ is the normalization constant, $\mathrm{B}=0.5\left(\mathrm{~m}_{0} / \sigma_{L}\right)^{2}$.

Fig. 5 shows the distribution of band gaps $\left(\mathrm{P}\left(\mathrm{E}_{G}\right)\right.$ when the energy upshift is given for $\mathrm{X}=1.4$ and $\mathrm{X}=2$. For the Lognormal case, we see that $\mathrm{P}\left(\mathrm{E}_{G}\right)$ peaks close to the band gap of c-si.

Equations 8, 12 and 13 are used to calculate $\alpha(E)$ for each incident energy $\mathrm{E}=$ $\hbar \omega$.

$\alpha(E)$ for a Gaussian distribution of sizes is given by

$$
\left.\alpha^{G}(E)=\alpha_{0} \int_{0}^{E_{b}}\left(E-E_{G}\right)^{-W}\left(E_{G}-E_{g}\right)^{(\gamma-X-3) / X} \exp \left[-A\left(\left(\frac{E_{G 0}-E_{g}}{E_{G}-E g}\right)^{1 / X}\right)-1\right)^{2}\right] d E_{G}
$$

where $\alpha_{0}$ is a normalization constant .

For a Lognormal distribution of sizes $\alpha(E)$ is

$\alpha^{L}(E)=\beta_{0} \int_{0}^{E_{b}}\left(E-E_{G}\right)^{-W}\left(E_{G}-E_{g}\right)^{((\gamma-X-2) / X)} \sigma_{L}^{-1} \exp \left[-B\left(\frac{\ln \left(C / E_{G}-E_{g}\right)^{1 / X}}{\ln \left(C / E_{G 0}-E_{g}\right)^{1 / X}}-1\right)^{2}\right] d E_{G}$ 
where $\beta_{0}$ is a normalization constant.

Equations (14) and (15) are used to simulate various possible absorption processes .

\section{RESULTS OF THE SIMULATION}

In this section, we present the results for the absorption co-efficient as a function of energy for different cases on the basis of the above model. The values of various exponents for different physical situations are summarized in Table I.

(1) Direct band gap

Figure 6 shows $\alpha(\hbar \omega)$ vs $\hbar \omega$ for a direct band gap material where $\mathrm{k}$ is conserved in the optical transition and no size dependence of the oscillator strengths $(\gamma=0)$ is considered (cases 1 and 3 and cases 5 and 7 in Table 1 ). To facilitate comparison between various cases, we have normalized the absorption to its peak value in each case. We see from the figure that the absorption tends to fall off at high energy ( in sharp contrast with the experimental data ). These results of the simulation can be easily understood as follows.

For a $1 \mathrm{D}$ system, the JDOS has a singularity at $\mathrm{E}=E_{G}$ and falls off for $\mathrm{E}>$ $\mathrm{E}_{G}$. The dominant contribution (to each $\alpha(E)$ ) is hence only from a particular set of nanostructures with band gap $E_{G}$ where $\mathrm{E}=E_{G}$ and all other $E_{G} \mathrm{~s}$ have almost no contribution. So the simulated $\alpha(E)$ vs E plot mimics $\mathrm{P}\left(E_{G}\right)$. We hence conclude from the simulation and the experimental data that PS is not a direct band gap material and we will not consider this case further.

(2) Indirect band gap

We now consider the case when momentum $(\mathrm{k})$ is not conserved for the optical transition. Here we discuss two cases - size independent oscillator strengths $(\gamma=0)$ and size dependent oscillator strengths ( assuming $\gamma=6$ ). 
We first consider the situation where the oscillator strength has no size dependence. Figure 7 (curves (a) and (c)) is a plot of the calculated $\alpha$ vs $\hbar \omega$ for a Gaussian distribution of sizes (case 2 and 4 of table I ). Figure 8 (curves (a) and (c)) is a plot of the calculated $\alpha$ vs $\hbar \omega$ for a Lognormal distribution of sizes (case 6 and 8 of Table I). Also shown in the figures is a plot of the experimental curve for a p type PS. (The dip in $\alpha$ near $2.2 \mathrm{eV}$ in the experimental curve is an artifact related to a filter change). To facilitate comparison, the calculated $\alpha$ is scaled to match the experimental curve near $3 \mathrm{eV}$. We see from the plots $7(\mathrm{a})$ and $7(\mathrm{c})$, that $\alpha$ initially increases with energy and then saturates - at variance with the experimental results. We understand this result qualitatively as follows. In section I, we have already mentioned that the JDOS is a constant independent of energy for $\hbar \omega<\mathrm{E}_{G}$ and zero for $\hbar \omega>\mathrm{E}_{G}$. Hence for $\hbar \omega>E_{G}$, a given nanostructure contributes equally at all energies to $\alpha$. However $\mathrm{P}\left(\mathrm{E}_{G}\right)$ falls off as $\mathrm{E}_{G}$ increases. Nanostructures having a band gap $\left(E_{G}\right)$ above a certain cut off in energy (in the high energy tail of the distribution) do not contribute significantly to $\alpha(\hbar \omega)$ (as $\mathrm{P}\left(\mathrm{E}_{G}\right)$ is very small). This gives rise to the saturation of $\alpha(E)$ in figures $7(\mathrm{a})$ and $7(\mathrm{c})$. This saturation is more obvious in figures $8(\mathrm{a})$ and $8(\mathrm{c})$, because $\mathrm{P}\left(\mathrm{E}_{G}\right)$ falls off very rapidly for a Lognormal distribution of sizes. These results also do not agree with the experimental data.

The situation is not remedied even if we include higher excited states $\left(\mathrm{n}_{x}=2\right.$, $\mathrm{n}_{y}=1$; etc.) in the energy range considered in our calculation. Based on the above discussion, we see that for $\alpha$ to increase with $\hbar \omega$, it is necessary to increase the contribution to $\alpha$ from the nanostructures in the high energy region of $\alpha(E)$. This is possible if the oscillator strength is enhanced for the nanostructures with large $E_{G}$ ( smaller sizes).

We now consider the case when $\mathrm{F}\left(E_{G}\right)$ varies as $f / d^{\gamma}$. Figure 7 (curves (b) and (d) for case 2 and 4 in Table I )and figure 8 (curves (b) and (d) for case 6 and 8 Table I ) are plots of $\alpha(\hbar \omega)$ vs $\hbar \omega$ for $\gamma=6$. For the Gaussian distribution that we have chosen, we see that the absorption still saturates although the onset of saturation moves to slightly higher energy. This is a measure of the contribution of the (high energy) tail of $P\left(E_{G}\right)$ for the Gaussian case to the total absorption. For the Lognormal distibution, we see from 
figure 8 (curves $(\mathrm{b})$ and $(\mathrm{d}))$ that $\alpha(\hbar \omega)$ vs $\hbar \omega$ now qualitatively looks like the experimental curve.

From a comparison of figures 7 and 8, we find that in order to get the absorption co-efficient to increase with incident energy, it is important that the contributions to $\alpha$ from the low energy regions of $\mathrm{P}\left(E_{G}\right)$ do not dominate the optical absorption at large $\hbar \omega$. This is also satisfied if the distribution of band gaps $\mathrm{P}\left(\mathrm{E}_{G}\right)$ is sufficiently broad. We illustrate this in Fig.9 where we plot the calculated value for $\alpha$ for a Gaussian distribution of band gaps with the mean energy at $2.25 \mathrm{eV}$ and a variance of 0.75 $\mathrm{eV}$. Hence we find that the nature of the band gap distribution $P\left(E_{G}\right)$ plays a crucial role in determining the shape of the absorption spectrum .

We conclude that to properly account for the optical absorption in PS we need to assume

(1) PS is a pseudo 1D indirect band gap semiconductor.

(2) The oscillator strength is size dependent and is given by a power law $f / d^{\gamma}$.

(3) The distribution $P\left(E_{G}\right)$ also plays an important role . Assuming a Lognormal distribution of the nanostructures diameters in porous silicon seems to naturally account for the absorption spectrum.

All these considerations are in general agreement with results of luminescence experiments on PS .The low temperature luminescence of PS excited near the peak of the broad luminescence spectrum exhibits steps which have been associated with the characteristic TO mode of c-silicon ${ }^{(23)}$. More recently ${ }^{(18)}$ well resolved peaks corresponding to the TO mode of Si have been reported for luminescence of PS suggesting that PS is an indirect band gap semiconductor. The observed dominant zero phonon component of luminescence constitutes direct evidence for the enhancement of the oscillator strength due to strong quantum confinement at smaller sizes ${ }^{(18,23,24)}$.

We now comment on the validity of the procedure for obtaining the band gap of PS from $\sqrt{\alpha \hbar \omega}$ vs $\hbar \omega$. Figure 10 is a simulated plot of $\sqrt{\alpha \hbar \omega}$ vs $\hbar \omega$ ( case 8 in Table I ) for three different values of $\gamma$ and the experimental data on p type sample. First, we note 
that the intercept of the linear portion of each of the simulated curves is neither coincident with the peak position ( near $1.3 \mathrm{eV}$ ) of the corresponding band gap distribution ( see figure 5 ) nor close to the mean band gap energy $E_{0}=2.25 \mathrm{ev}$. So we see that it is very difficult to relate this intercept with such physical quantities .Therefore, it is not obvious how to interpret the intercept with some kind 'effective band gap'. We also note that the intercept depends on the choice of $\gamma$. We hence believe that the intercept does not have the simple interpretation of a band gap as defined for a homogeneous system. We would like to point out that for such a heterogeneous system the gap can be interpreted only in an operational sense like $\mathrm{E}_{03}$ or $\mathrm{E}_{04}$ - depending on the value of the absorption coefficient (after correcting for the sample porosity).

Finally we try to understand the temperature dependence of the band gap. To explain the rigid shift of the absorption curve with temperature (to lower $\alpha$ ), we argue that $E_{G}$ of each of the nanostructures follows a relation $E_{G}(\mathrm{~T})=E_{G 0}-\gamma T$, where $E_{G 0}$ is the band gap at zero degree Kelvin. We assume that the change of the band gap with temperature is a consequence, like in bulk semiconductors, of the electron -phonon interaction and take $\gamma=0.5 \mathrm{meV} / \mathrm{K}$. In our model, changing temperature is equivalent to changing the value of $\mathrm{C}$ in the expression that determines the energy upshift $\Delta E$. Figure 11 is a plot of $\ln (\alpha)$ vs $\hbar \omega$ for three different values of $\mathrm{C}$. We see that the absorption curves are rigidly shifted vertically with respect to each other ${ }^{(10)}$ as also seen in figure 2 . We argue that this provides a natural explanation for the observed temperature dependence of $\alpha$.

\section{CONCLUSIONS}

In this paper we have studied the absorption in free standing thin films of PS .

We have calculated the absorption coefiicient as a function of energy assuming that PS can be modelled as a pseudo 1D system having a distribution of band gaps . From our calculations, we show that it is neccessary to look at PS as an indirect band gap 
material. To satisfactorily account for the absorption, we need to invoke that the oscillator strengths has a size dependence. We also show that a Lognormal distribution

of diameters of the nanostructures that make up PS appears to account for the measured absorption spectrum of p PS .

We argue that one can not use eq.1 to obtain the band gap of such low

dimensional materials like porous silicon. We also explain the temperature dependence of the absorption in PS on the basis of our model.

We have also shown that porosity can be inferred non-destructively from a transmission measurement in the region of low absorption .

\section{ACKNOWLEDGEMENT}

The authors like to thank Prof. B.M.Arora and Prof. Rustagi for valuable suggestions . S.D thanks Sandip Ghosh, Anver Aziz, M.K.Rabinal and Biswajit Karmakar for useful discussions and A.D.Deorukhkar , R.B.Driver , V.M.Upalekar and P.B.Joshi for their help . 


\section{REFERENCES}

${ }^{1}$ L.T. Canham, Appl. Phys. Lett.,57, 1046 (1990).

${ }^{2}$ A.G. Cullis, L.T. Canham, Nature, 353, 335 (1991).

${ }^{3}$ V. Lehmann, U. Gosele, Appl. Phys. Lett., 58, 856 (1991).

${ }^{4}$ Reuben T. Collins, Philippe M. Fauchet, Michael A. Tischler, Phys. Today, January, 24 (1997).

${ }^{5}$ Philippe M. Fauchet, Semiconductors and Semimetals, Academic Press, Vol. 49, 205 (1998).

${ }^{6}$ A.G. Cullis, L.T. Canham, P.D.J. Calcott, J. Appl. Phys., 82, 909 (1997).

${ }^{7}$ Yoshihiko Kanemitsu, Phys. Rep., 263, 1 (1995).

${ }^{8}$ George C. John, Vijay A. Singh, Phys. Rep., 263, 93 (1995).

${ }^{9}$ G. Bomchil, A. Halimaoui, I. Sagnes, P.A. Badoz, I. Berbezier, P. Perret, B. Lambert, G. Vincent, L. Garchery and J.L. Regolini, Appl. Surf. Sci., 65/66, 394 (1993).

${ }^{10}$ D. Kovalev, G. Polisski, M. Ben-Chorin, J. Diener and F. Koch, J. Appl. Phys., 80, 5978 (1996).

${ }^{11}$ I.Sagnes, A.Halimaoui, G.Vincent, and P.A.Badoz, Appl.Phys.Lett., 62, 1155 (1993).

${ }^{12}$ A. Kux, M. Ben-Chorin, Phys. Rev. B, 51, 17535 (1995).

${ }^{13}$ J.I. Pancove, Optical processes in semiconductors, Prentice Hall (1971), Chapter - 3.

${ }^{14}$ J. von Behren, T. van Burren, M. Zacharias, E.H. Chimowitz, P.M. Fauchet, Sol. State. Comm., 105, 317 (1998).

${ }^{15}$ Mark S. Hybertsen, Phys. Rev. Lett., 72, 1514, (1994).

${ }^{16}$ J.B. Khurgin, E.W. Forsythe, G.S. Tompa, B.A. Khan, Appl. Phys.Lett, 69, 1241 (1996). 
${ }^{17}$ G.D. Sanders, Yia-Chung Chang, Phys. Rev. B, 45, 9202 (1992).

${ }^{18}$ D. Kovalev, H.Heckler, M. Ben-Chorin, G.Polisski, M.Schwartzkopff, F.Koch, Phys. Rev. Lett., 81, 2803 (1998).

${ }^{19}$ H. Cramer, Mathematical methods of Statistics, Princeton University Press, (1946), Chapter 17 .

${ }^{20}$ A.J. Read, R.J. Needs, K.J. Nash, L.T. Canham, P.D.J. Calcott and A. Qteish, Phys. Rev. Lett., 69, 1232 (1992).

${ }^{21}$ C.D.Delerue, G.Allan, M.Lannoo , Phys. Rev. B, 48, 11024 (1993).

${ }^{22}$ V. Ranjan, Vijay A. Singh, George C. John, Phys. Rev. B, 58, 1158 (1998).

${ }^{23}$ P.D.J.Calcott, K.J.Nash, L.T.Canham, M.J.Kane, D.Brumhead, J.Phys:Cond.Matt. , 5, L91 (1993). 


\begin{tabular}{|c|c|c|c|c|c|}
\hline \hline \multicolumn{3}{|c|}{ Gaussian distribution in $\mathrm{d}$} & \multicolumn{3}{|c|}{ Lognormal distribution in $\mathrm{d}$} \\
\hline Case & Value of X & Nature of Transition & Case & Value of X & Nature of Transition \\
\hline 1. & 2.0 & Direct & 5. & 2.0 & Direct \\
\hline 2. & 2.0 & Indirect & 6. & 2.0 & Indirect \\
\hline 3. & 1.4 & Direct & 7. & 1.4 & Direct \\
\hline 4. & 1.4 & Indirect & 8. & 1.4 & Indirect \\
\hline \hline
\end{tabular}

TABLE I. These physical situations we have considered in the model for the optical absorption in porous silicon .For direct transitions $\mathrm{W}=0.5$ and for indirect transitions $\mathrm{W}$ $=0$. The value of $\gamma$ is taken as 6.0 for size dependent oscillator strengths and zero for the size independent case. 


\section{FIGURES}

FIG. 1. Porosity calculated ( using eq. 7 ) from the transmission curves ${ }^{(9,14)}$ are plotted against the corresponding gravimetrically determined values of porosity . The points fall nearly on a straight line of slope one. (a) data from ref. 9 and (b) data from ref. 14 .

FIG. 2. Normalized transmission spectrum for (a) $\mathrm{p}^{+}$and (b) p type porous silicon. The onset of low absorption is at smaller wavelength for $\mathrm{p}$ si than that of $\mathrm{p}^{+}$si.

FIG. 3. Temperature dependence of the absorption spectrum of p type porous silicon . (a) $300 \mathrm{~K}$ and (b) $100 \mathrm{~K}$.

FIG. 4. Size distribution of the column widths . (a) For a Gaussian and (b) for a Lognormal distribution of widths, having $\mathrm{d}_{0}=30$ Åand $\sigma=4 \AA$.

FIG. 5. Band gap distribution for corresponding size distribution given in figure 4. (a) and (b) are for Gaussian distribution for $\mathrm{X}=2$ and 1.4 respectively.(c) and (d) are for the Lognormal distribution for $\mathrm{X}=2$ and 1.4 respectively.

FIG. 6. Simulated $\alpha(E)$ vs E plot for direct transitions $(\gamma=0)$. Plots (a) and (b) are for the cases (1) and (3) in Table I . Plots (c) and (d) are for the cases (5) and (7) in Table I.

FIG. 7. Simulated $\alpha(E)$ vs E plot for indirect transitions .(a) and (b) correspond to case (2) in table I for $\gamma=0$ and 6 respectively .(c) and (d) correspond to case (4) in table I for $\gamma=0$ and 6 respectively .(e) Measured $\alpha(E)$ vs E plot for p type PS.

FIG. 8. Simulated $\alpha(E)$ vs E plot for indirect transitions .(a) and (b) correspond to case (6) in table I for $\gamma=0$ and 6 respectively .(c) and (d) correspond to case (8) in table I for $\gamma=0$ and 6 respectively . (e) Measured $\alpha(E)$ vs E plot for p type PS.

FIG. 9. (a)Simulated $\alpha(E)$ vs E plot for broad gaussian distribution of band gaps $\left(\mathrm{E}_{0}=2.25\right.$ $\mathrm{eV}$ and $\sigma_{E}=0.75 \mathrm{eV}$ ) with indirect transitions, $\mathrm{X}=1.4$ and $\gamma=6$. (b) Measured $\alpha(E)$ vs $\mathrm{E}$ plot for $\mathrm{p}$ type PS. 
FIG. 10. Simulated plots of $(\alpha \hbar \omega)^{1 / 2}$ vs $\hbar \omega$ for (a) $\gamma=5$, (b) $\gamma=5.5$, (c) $\gamma=6.0$ and (d) corresponding plot for the experimental data on p type PS .

FIG. 11. Simulated $\log (\alpha(E))$ vs E plots for various temperatures ( for case 8 with $\gamma=6$ ). (a) $\mathrm{C}=126=C_{0}$, (b) $\mathrm{C}=1.25 C_{0}$, (c) $\mathrm{C}=1.5 C_{0}$. Curves are rigidly shifted vertically from one another. 
Figure 1 .

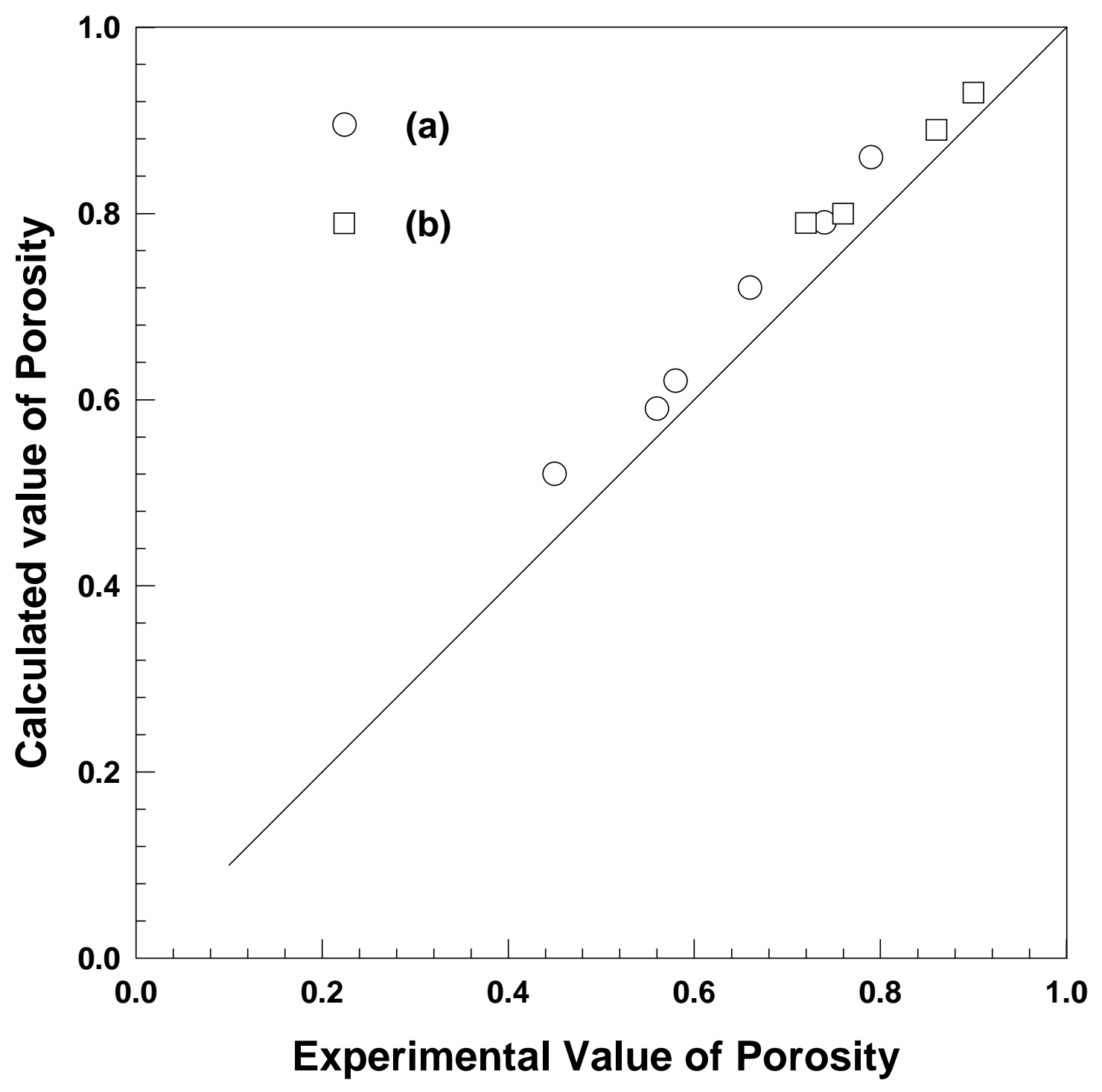


Figure 2 .

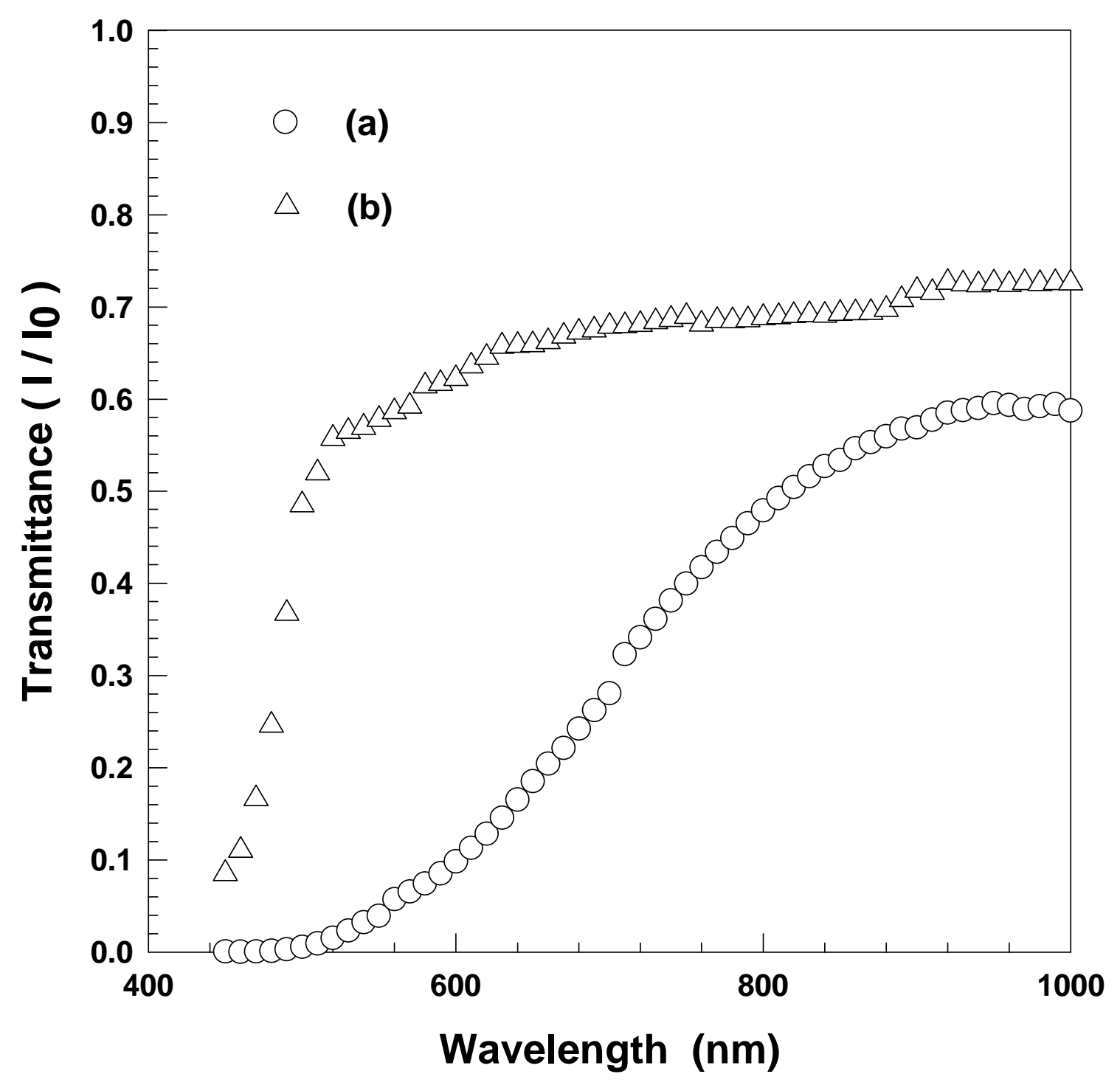


Figure 3 .

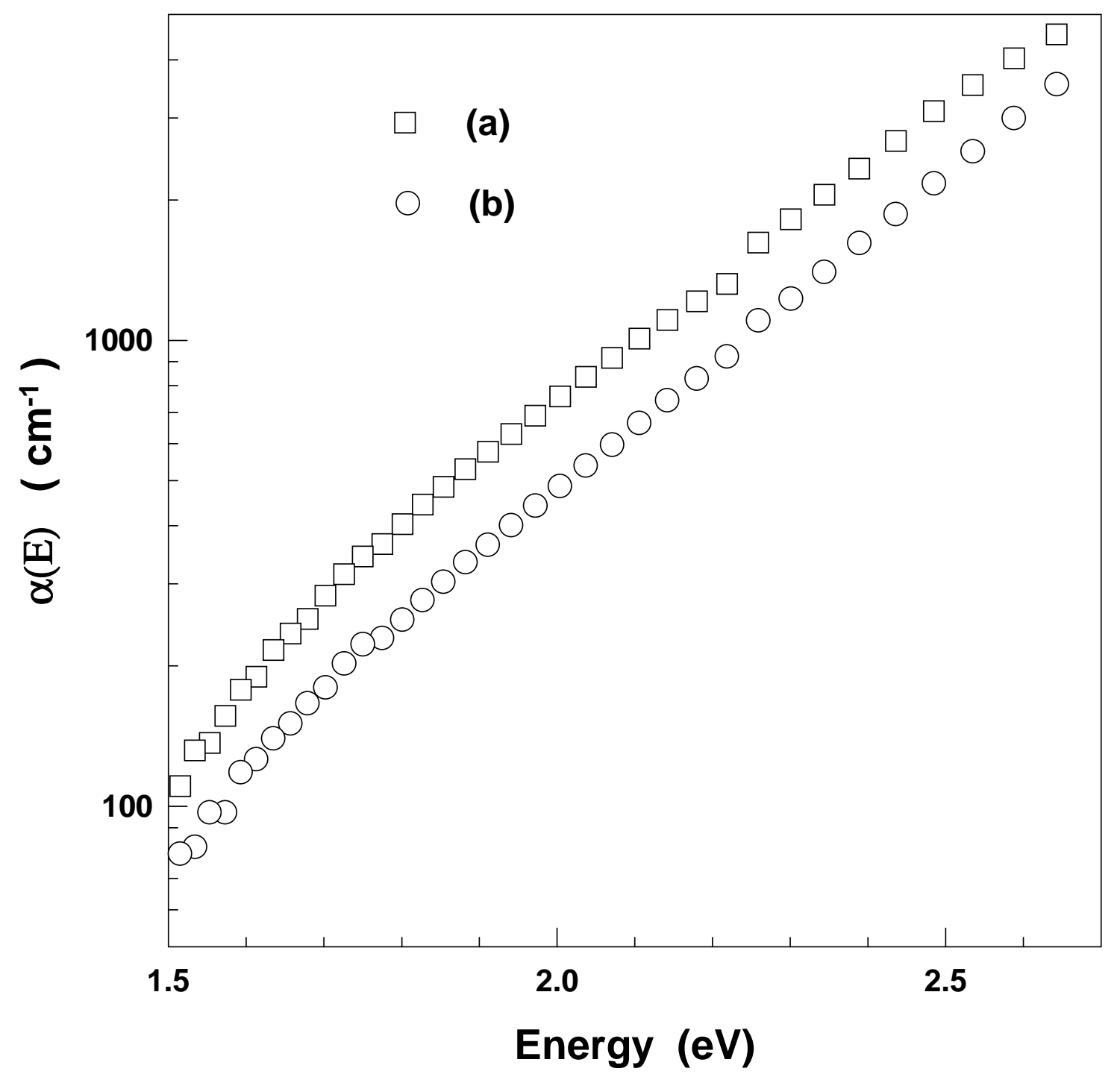


Figure 4 .

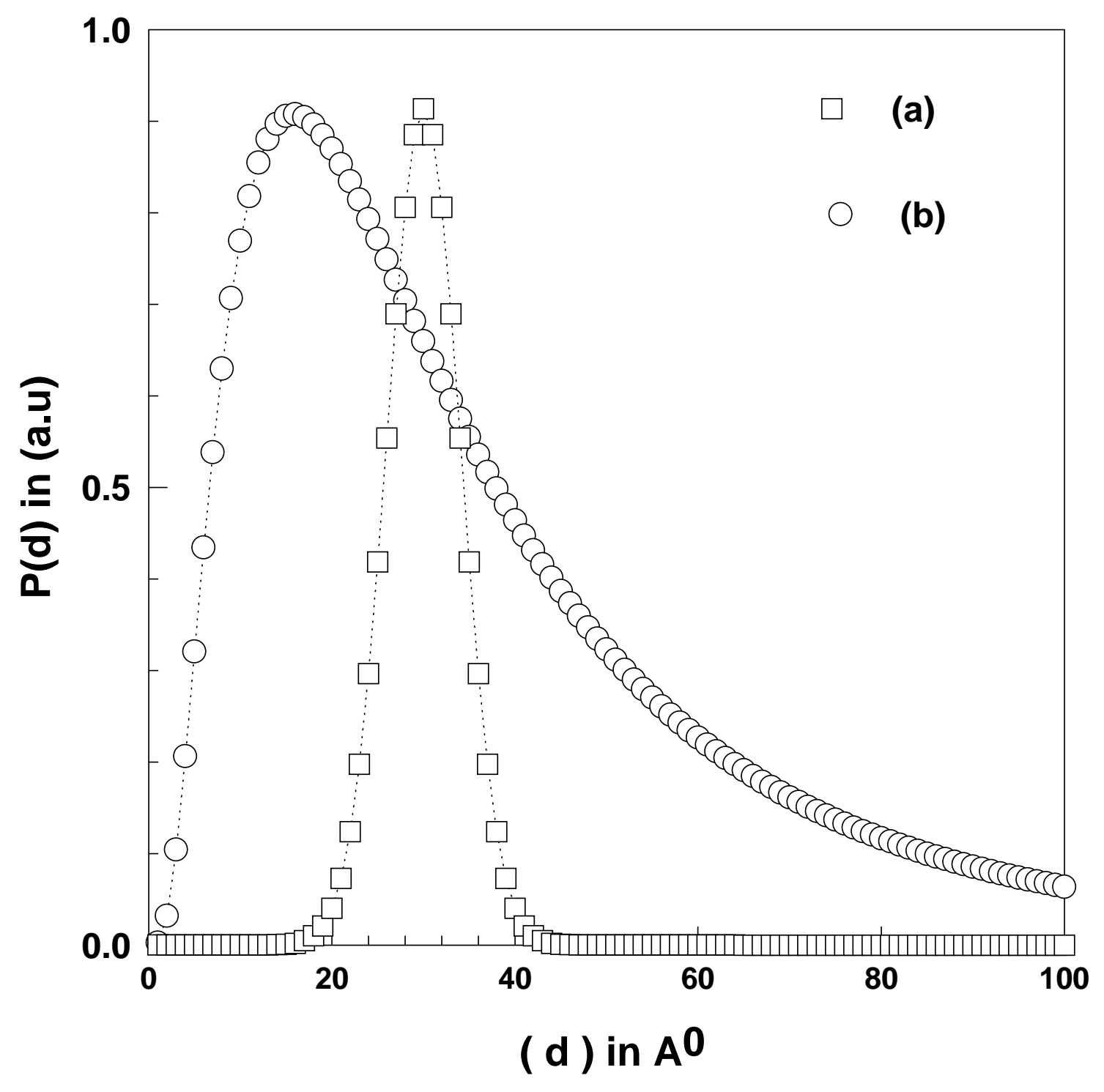


Figure 5.

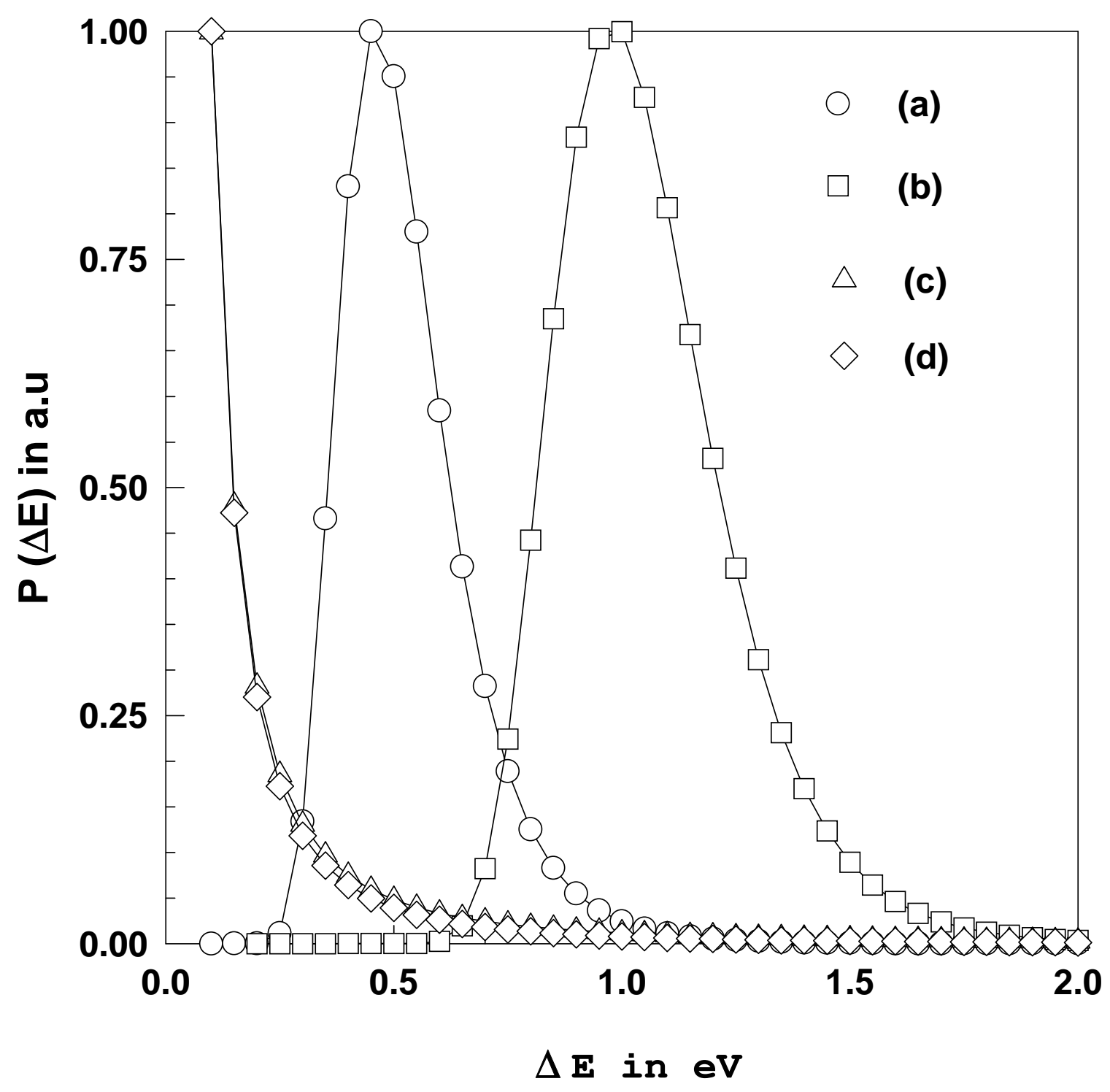


Figure 6.

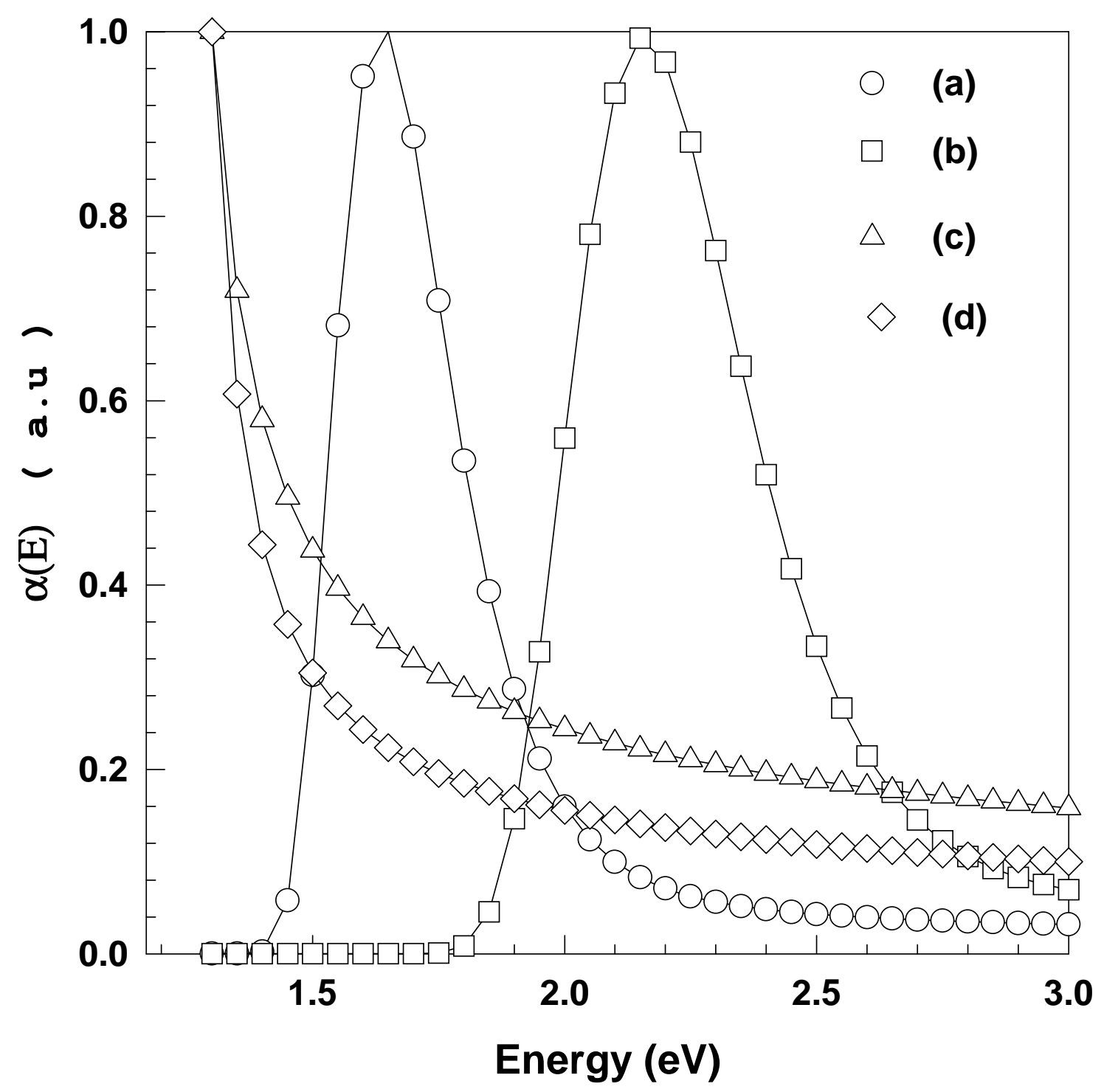


Figure 7.

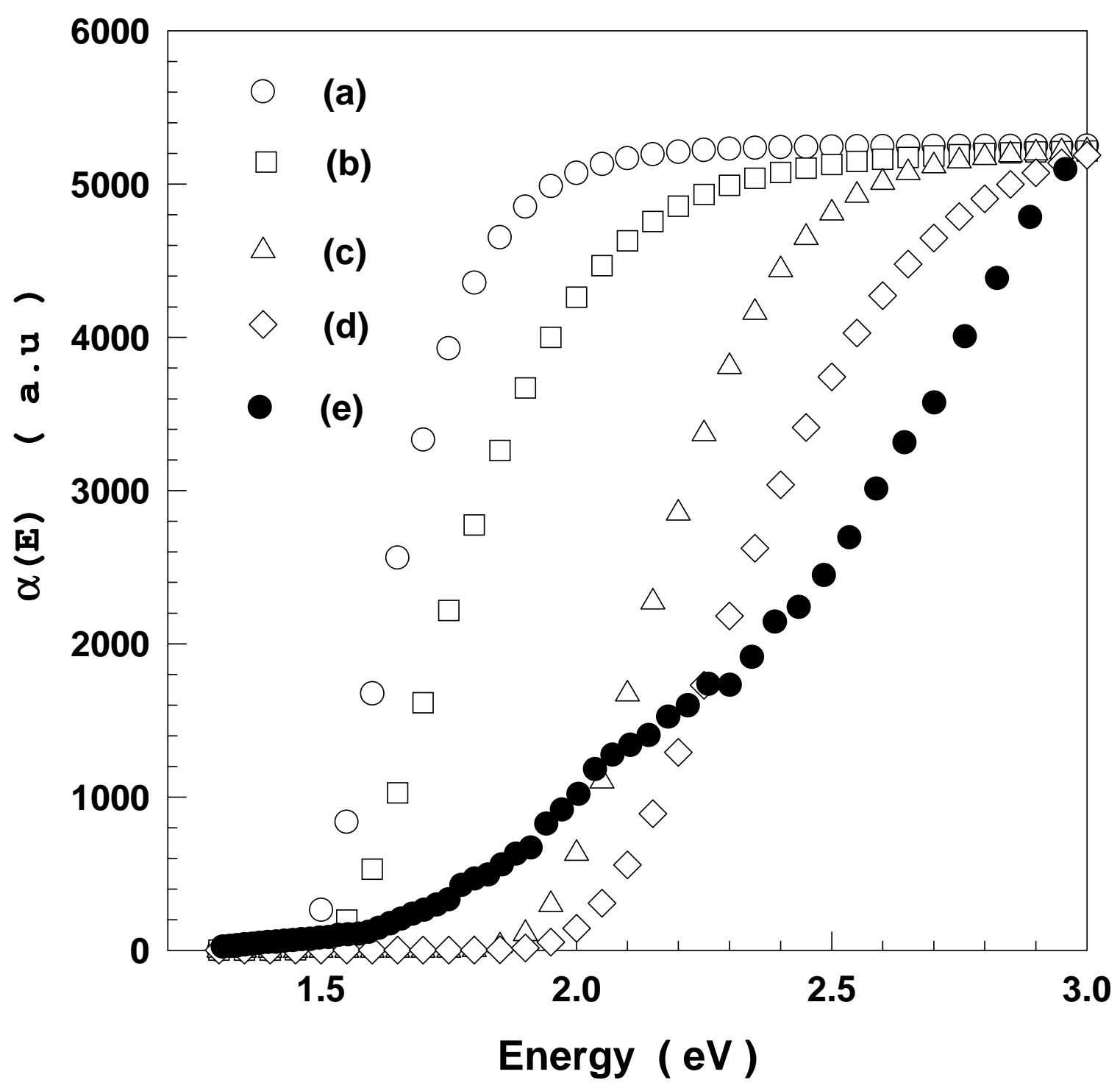


Figure 8.

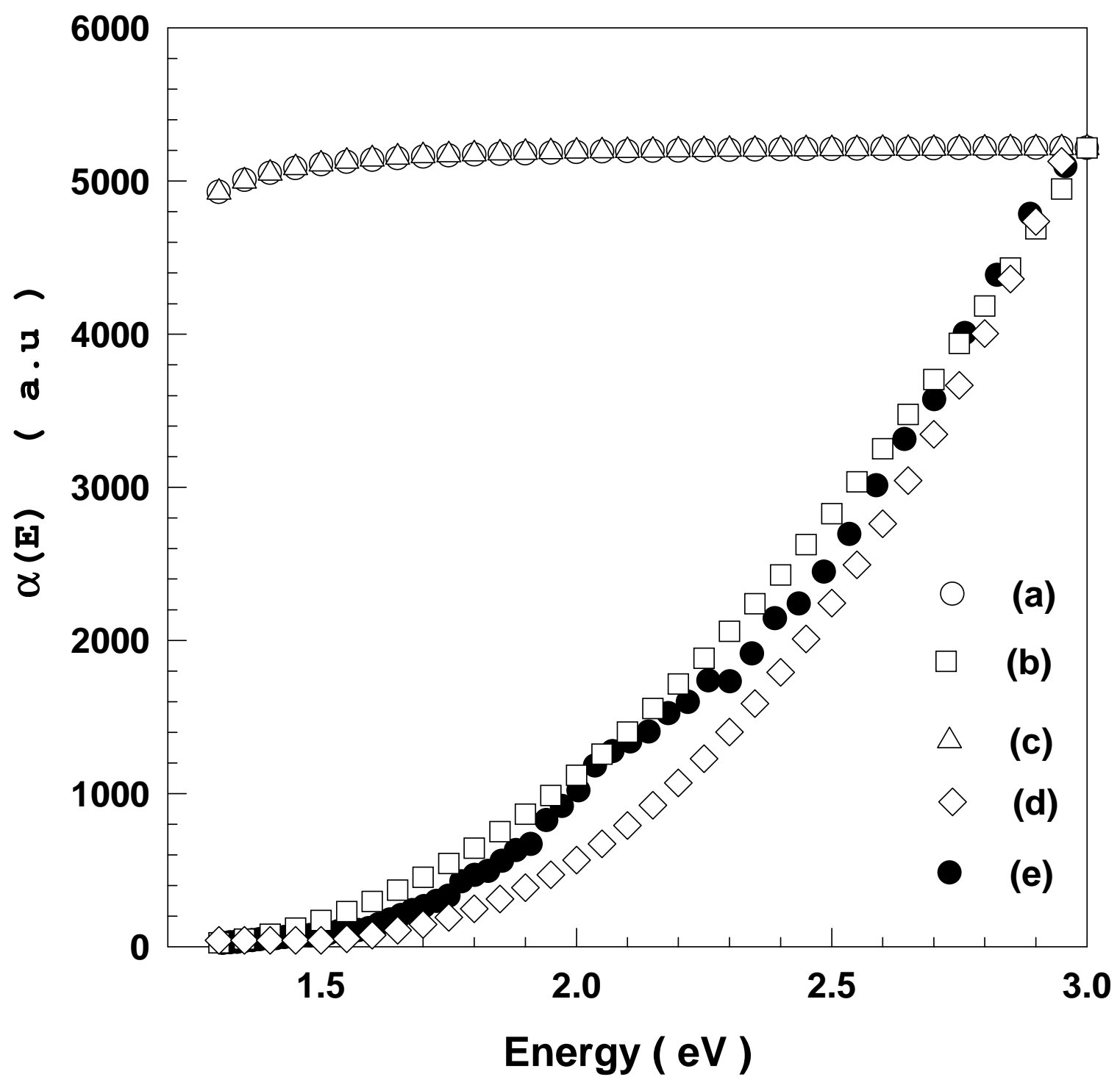


Figure 9.

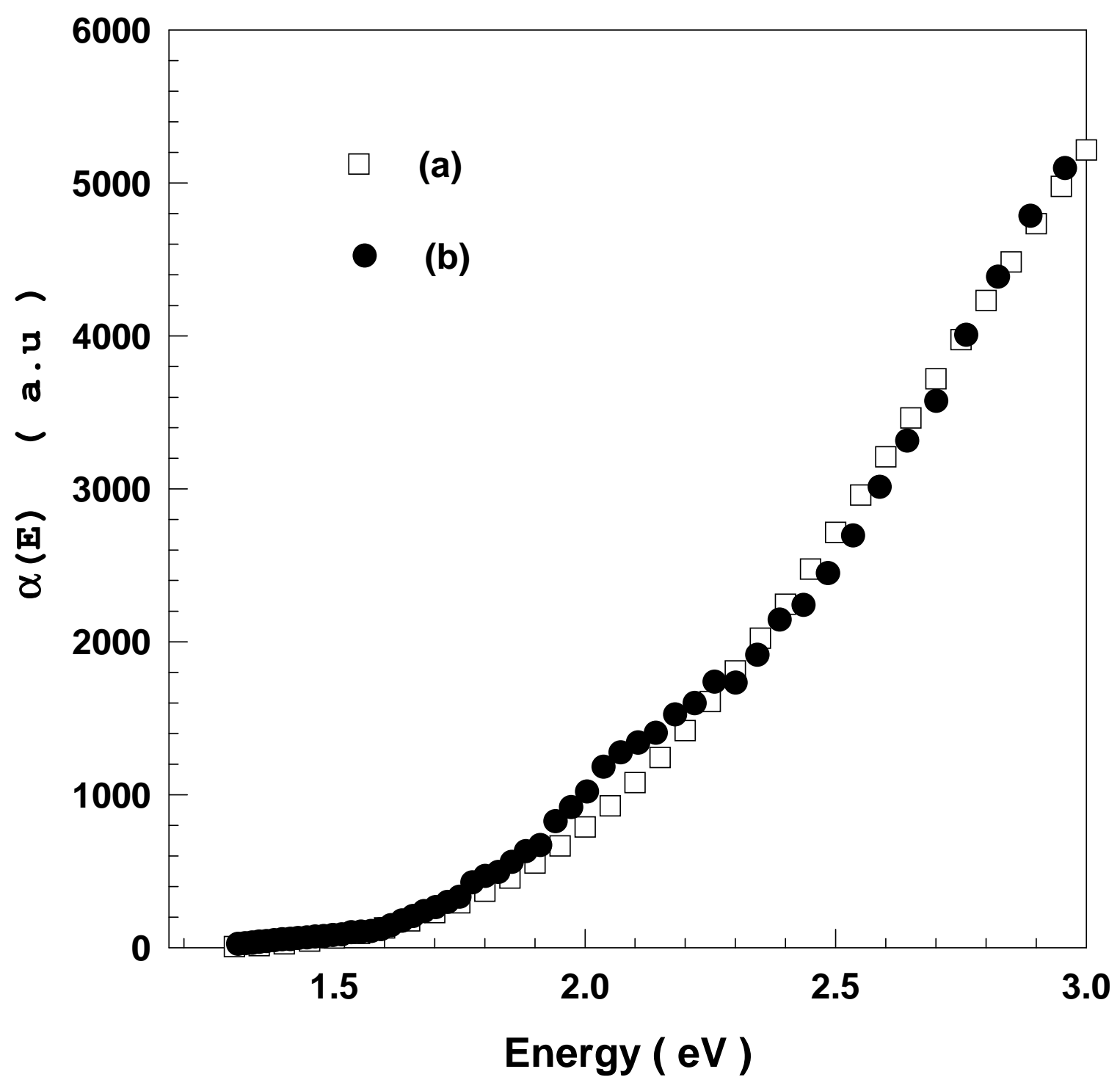


Figure 10.

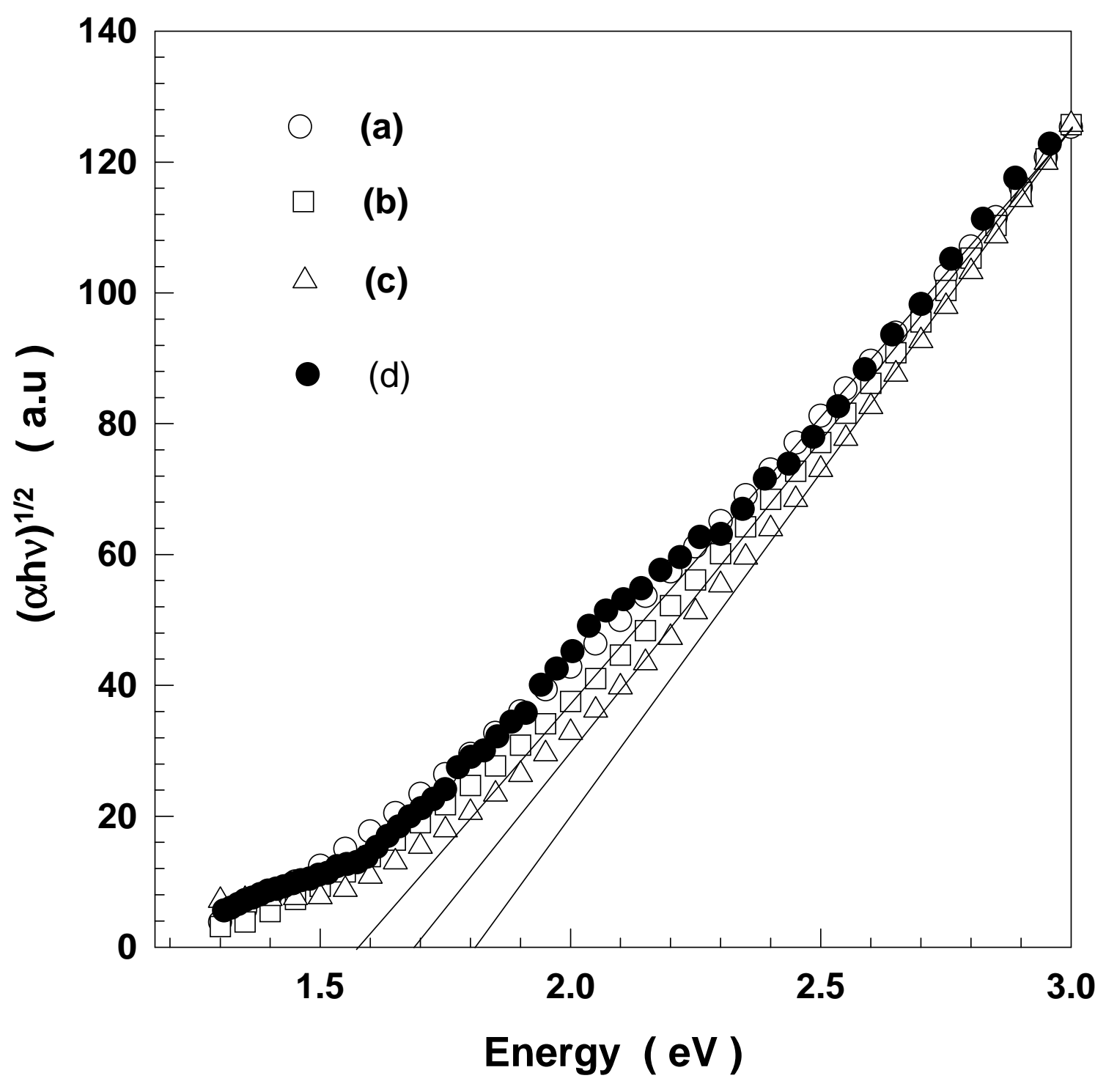


Figure 11.

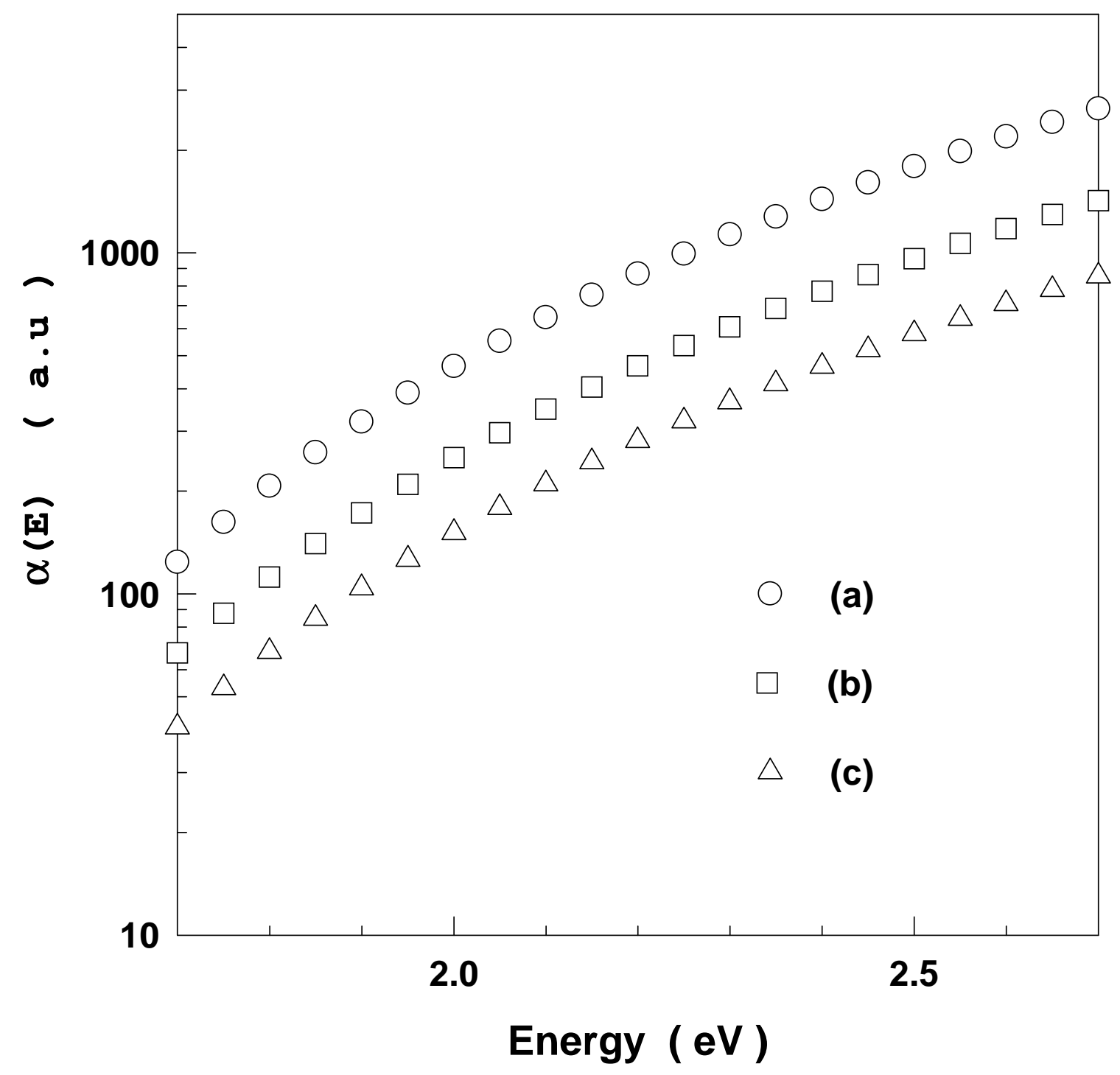

